\title{
El modelo porcino en la cirugía experimental del trasplante renal
}

\author{
Bestard Vallejo JE, Raventós Busquets CX, Celma Doménech A, Rosal Fontana M*, \\ Esteve $\mathrm{M}^{*}$, Morote Robles J. \\ Servicio de Urología y *Estabulario del Institut de Recerca. Hospital Universitario Vall d'Hebron. \\ UAB. Barcelona.
}

Actas Urol Esp. 2008;32(1):91-101

\section{RESUMEN}

EL MODELO PORCINO EN LA CIRUGÍA EXPERIMENTAL DEL TRASPLANTE RENAL

Introducción y objetivos: La extracción renal laparoscópica de donante vivo se está convirtiendo en una técnica cada vez más habitual a pesar de su lenta curva de aprendizaje. En enero de 2006 se puso en marcha en nuestro centro un protocolo de autotrasplante en modelo porcino que pretendía evaluar diferencias entre la extracción por vía abierta y por vía laparoscópica, y que permitía así mismo entrenar a los residentes en la realización de estas técnicas.

Material y métodos: Hasta día de hoy hemos llevado a cabo un total de 25 experimentos, de los cuales 7 han sido sometidos a extracción por vía laparoscópica (28\%) y 18 por vía abierta (72\%), si bien sólo el $44 \%$ de los animales ha sobrevivido hasta el final del protocolo.

Resultados: En este trabajo se detalla la forma de iniciar una experiencia de estas características, se analizan las complicaciones que se han encontrado y se hace una revisión de la literatura en cuanto al manejo de estos animales.

Conclusiones: La cirugía experimental en modelo porcino se ha convertido en nuestro centro en una herramienta fundamental para la formación del residente, y podría fácilmente ser adaptada por otros hospitales.

Palabras clave: Modelos experimentales. Trasplante renal.

\section{ABSTRACT}

PIG MODEL IN EXPERIMENTAL RENAL TRANSPLANT SURGERY

Introduction and objectives. Living donor laparoscopic renal procurement is becoming a first-line technique unless a show-learning curve. January 2006 we implement an experimental pig-kidney transplant model with the objective of evaluating differences between open and laparosopic surgical techniques as well as giving a training-oportunity to the Residents in these alternatives.

Material and methods. We have completed 25 experiments 7 out of which were performed laparoscopically (28\%), 18 with conventional surgery (72\%). Only $44 \%$ of the animals have survived until the end of the process.

Results: This work evaluates different aspects on the implementation of this activity. Complications of the prothocol are analyzed. We review the literature on this topic.

Conclusions: Experimental Surgery in a porcine model has become in our Hospital a key-issue for Residents Training Program, and easily could be adapted to other Centers.

Keywords: Experimental models. Renal transplantation.

$\mathrm{E}^{\prime}$ creciente número de pacientes que se encuentran a la espera de trasplante renal, así como la progresiva disminución del número de donantes cadáver, está haciendo que cada vez se tienda más a considerar la extracción renal de donante vivo como una alternativa viable $y$, según los resultados publicados, con mejor supervivencia del injerto respecto a los de pacientes fallecidos ${ }^{1}$. 
Sin embargo, la morbilidad de la cirugía y las molestias tanto clínicas como estéticas que supone para el paciente la incisión de lumbotomía que habitualmente se practica, está favoreciendo que en muchos centros se opte por la cirugía laparoscópica como el abordaje de elección ${ }^{2}$.

En nuestro centro, si bien existe larga experiencia en lo que a trasplante renal se refiere, hace apenas un año que se está practicando la extracción renal de donante vivo de esta forma, siendo para el residente dificil aprender dicha técnica debido al hasta ahora reducido número de donantes vivos.

Desde el mes de enero de 2006, se practica un programa de cirugía experimental con modelo porcino mediante el cual se pretendía evaluar las diferencias de la extracción renal por vía abierta respecto a la vía laparoscópica. Estos experimentos permitirán, no sólo evaluar las ventajas e inconvenientes de ambas vias, sino también adiestrar a los residentes en las dos técnicas para poder reproducirlas posteriormente en humanos.

En este artículo se describe cómo se llevan a cabo tales procedimientos y las dificultades que hemos encontrado, asi como los resultados que hemos obtenido en cuanto a supervivencia y tiempos quirúrgicos. Pretende, en definitiva, ser una guía útil para aquellos centros interesados en poner en marcha un proyecto de caracteristicas similares.

\section{MATERIAL Y MÉTODOS}

En el mes de enero de 2006, se inició en nuestro centro un protocolo en modelo porcino, mediante el cual se sometía a los animales a autotrasplante renal con la extracción por vía abierta o por vía laparoscópica, sacrificándose a los 7 días de la intervención. Se evaluaron las diferencias tanto morfológicas como funcionales que conlleva uno u otro método. Dicho protocolo fue revisado y aceptado por el Comité Ético de Experimentación Animal del Hospital Universitario Vall d'Hebron.

\section{Animales}

El estudio se planteó inicialmente con 20 cerdos hembra híbridos entre Large White y Landrace, de 4 meses de edad, libres de patógenos específicos y de entre 30 y $35 \mathrm{~kg}$ de peso. Sin embargo, la alta mortalidad obtenida con estos animales derivó en que a partir del experimento 17 se decidiera practicar con cerdos macho, de la misma raza, peso y edad.

Las extracciones se practicaban alternativamente por una y otra vía. Si un cerdo no sobrevivía los 7 días tras la intervención se consideraba como no valido para el experimento y se practicaba un nuevo experimento por la misma vía que el anteriormente fallecido.

\section{Preoperatorio}

Los animales llegaban al estabulario una semana antes para su aclimatación y comprobación del estado sanitario, permaneciendo en ayunas desde 12 horas antes del primer tiempo quirúrgico a excepción del agua.

A la mañana siguiente los veterinarios de nuestro centro se encargaban de llevar a cabo una premedicación del animal mediante la inyección intramuscular de zoletil (tiletamina/zolacepam) $(3 \mathrm{mg} /$ $\mathrm{kg})$, xilacina $(2 \mathrm{mg} / \mathrm{kg})$ y atropina $(0,01 \mathrm{mg} / \mathrm{kg})$. La consecución de la preanestesia se comprobaba mediante la abolición del reflejo de estación (imposibilidad del animal para permanecer de pie).

Tras 20 minutos el animal era transportado desde la corralina hasta el prequirófano, donde era convenientemente pesado, lavado y rasurado. Igualmente se le colocaba una vía periférica a nivel de una vena auricular de $20 \mathrm{G}$ y $2,5 \mathrm{~cm}$ de longitud que se fijaba con esparadrapo.

Se procedía a continuación a trasladar al animal a la mesa de quirófano, donde se le colocaba en posición de decúbito prono sobre una manta térmica para controlar la temperatura.

\section{Anestesia}

En un primer grupo de cerdos la inducción anestésica se inició con propofol ev a dosis de 2 $\mathrm{mg} / \mathrm{kg}$, llevando a cabo el mantenimiento también con propofol ev a dosis de 0,2-1,3 mg/ $\mathrm{kg} /$ min, comprobándose mediante el reflejo podal la consecución del máximo grado de anestesia.

Sin embargo, dada la alta tasa de mortalidad intraoperatoria relacionada con el despertar de los animales, se decidió a partir del experimento 17 llevar a cabo tanto la inducción, como el mantenimiento de la anestesia mediante inhalación de isofluorano al $2 \%$. 
Durante la intervención, la analgesia se llevaba a cabo con fentanilo ev a dosis de 1,5-3 mg/kg.

\section{Ventilación}

La preoxigenación se llevaba a cabo con mascarilla de Hall durante 5 minutos, momento en el cual, tras comprobarse un reflejo laringeo negativo, el anestesista procedía a la intubación con un tubo endotraqueal de $7 \mathrm{~mm}$. Si en el momento de la intubación se observaba un reflejo laríngeo positivo se administraba un bolus de propofol a dosis de $3 \mathrm{mg} / \mathrm{kg}$ (Fig. 1).

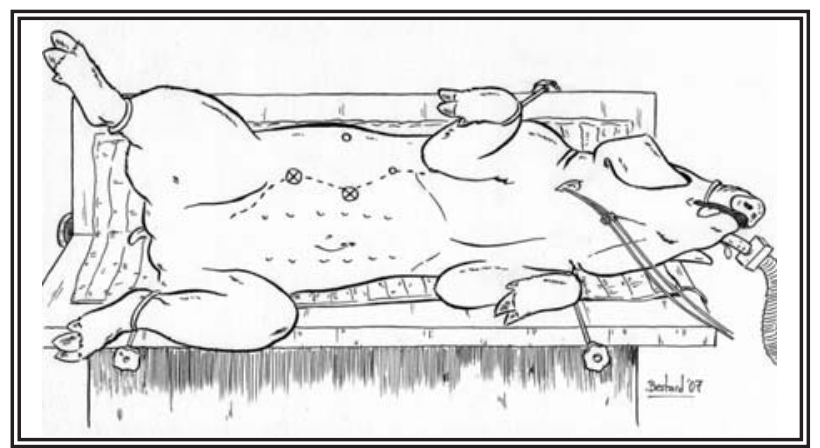

FIGURA 1. Disposición de los trócares y trayecto de laparotomía.

La ventilación mecánica se mantenía con el respirador veterinario Model 2000 de Hallowell EMC, manteniendo una fracción de inspiración de oxigeno (FiO2) de 100\% con una presión inspiratoria pico máxima (PIP max) de $20 \mathrm{~cm} \mathrm{H}_{2} \mathrm{O}$, un volumen corriente de $10-15 \mathrm{ml} / \mathrm{kg}$ y una presión positiva intermitente (PEEP) de $5 \mathrm{~cm}$ de $\mathrm{H}_{2} \mathrm{O}$.

\section{Canulación}

Una vez intubado, un residente procedía al entallado de la zona paratraqueal derecha, practicando en condiciones estériles una incisión de $10 \mathrm{~cm}$. y disección por planos hasta identificar la vena yugular y arteria carótida externas, que eran canuladas mediante un catéter de $2,1 \mathrm{~mm}$ de diámetro para el mejor manejo de la sueroterapia, obtención de presión venosa y arterial, así como extracción de muestras analíticas al inicio y final de la cirugía y en los días posteriores.

\section{Monitorización}

En todo momento el animal permanecía monitorizado mediante:
- Presiones centrales arteriales y venosas.

- Temperatura a través de un sensor intraesofágico.

- Capnografia.

- Pulsioximetría, mediante un pulisoxímetro colocado en la cola (y si estaba cortada, en el pabellón auricular o la lengua).

- Concentraciones espiradas de $\mathrm{CO}_{2}$.

- ECG, mediante 3 electrodos colocados en el tórax.

- Gasometrias arteriales al inicio de la cirugía y tras la reperfusión del injerto (Fig. 2).

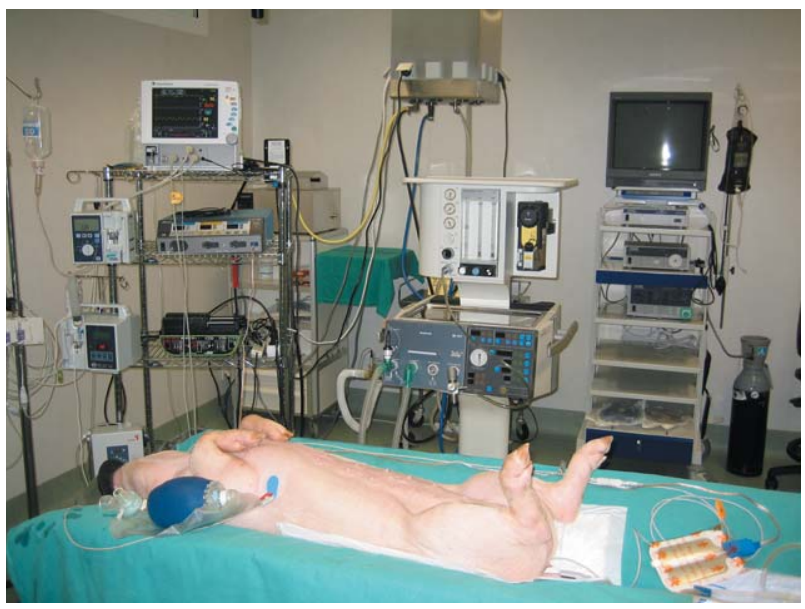

FIGURA 2. Animal en la mesa quirúrgica.

\section{Cirugia}

Extracción renal laparoscópica

1. Colocación de los animales en decúbito lateral izquierdo (se escogía el riño derecho por la mayor tendencia del derecho a presentar más de una vena renal, lo que hubiera dificultado la anastomosis).

2. Nuevo entallado y creación un neumoperitoneo mediante inyección de $\mathrm{CO}_{2}$ con aguja de Veress a nivel de la línea abdominal media, con una presión durante todo el acto de $15 \mathrm{~mm} \mathrm{Hg}$.

3. Colocación de un primer trocar de $10 \mathrm{~mm}$ en la línea axilar anterior a nivel de la última costilla, por el que se introducía una óptica con ángulo de 30 grados. Posteriormente colocación de tres trócares más, uno de $10 \mathrm{~mm}$. y otros dos de $5 \mathrm{~mm}$., en forma de rombo con el primero (Fig. 3).

4. Localización del uréter derecho, que era disecado hasta llegar al pedículo renal. El riñón no se desprendía de la cara posterior del peritoneo hasta el final del acto, dado que esto hubiera dificultado la disección del pedículo. 


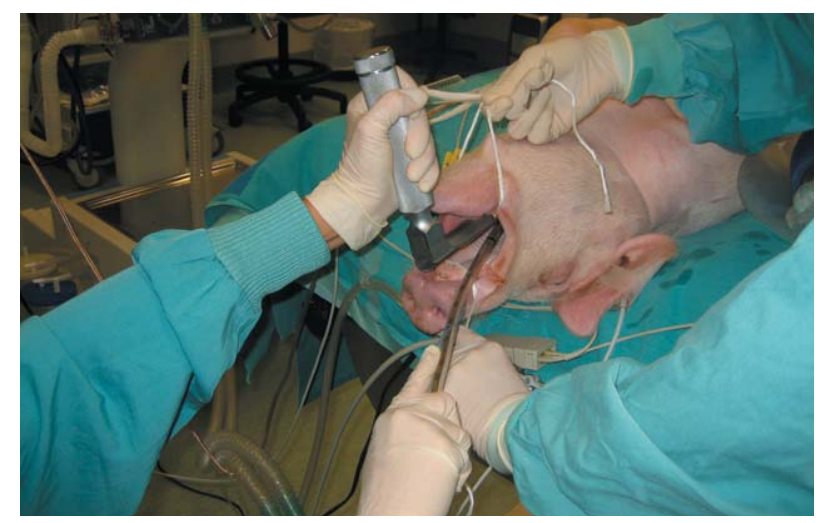

FIGURA 3. Intubación con tubo endotraqueal.

5. Disección de arteria y vena renales, ligadura con hemolocks o clips vasculares y sección de las mismas dejando la máxima longitud posible. El tiempo fijado de mantenimiento del neumoperitoneo era de 45 minutos (Fig. 4).

6. Colocación del animal en decúbito supino y laparotomía longitudinal siguiendo los orificios de los 3 trócares más mediales (Fig. 3).

7. Nefrectomía contralateral y obtención de una primera biopsia de ese riñón.

8. Colocación del riñón derecho en recipiente con hielo y perfusión arterial mediante un abbocath de $20 \mathrm{G}$ con solución de Wisconsin por gravedad hasta obtener líquido claro por la vena renal. El tiempo de isquemia caliente no debía ser superior a 15 minutos.

\section{Extracción renal vía abierta}

1. Colocación de los animales en decúbito supino, practicándose la laparotomía lateral mencionada anteriormente.

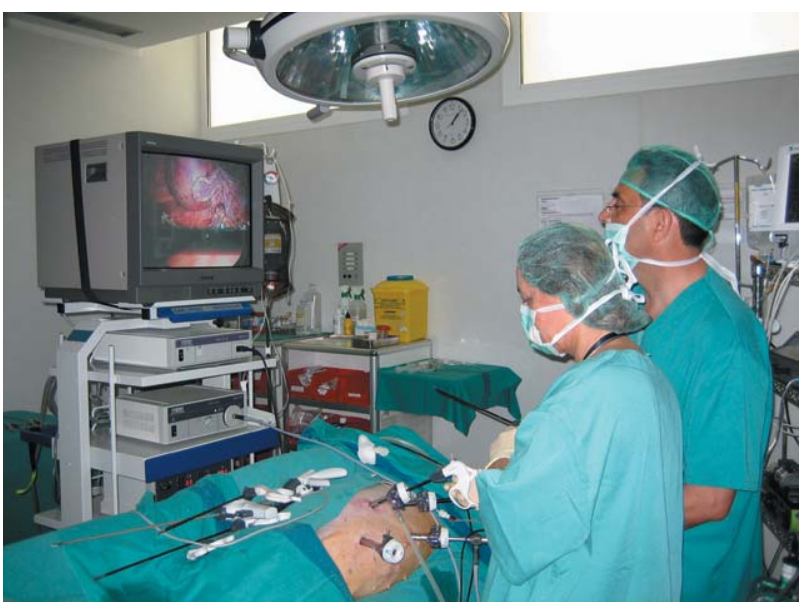

FIGURA 4. Disección laparoscópica del pedículo renal.
2. Disección del riñón derecho, sutura de los vasos a nivel distal y sección de los mismos.

3. Nefrectomía izquierda y obtención de biopsia de ese riñón.

4. Colocación del riñón derecho en recipiente con hielo y perfusión arterial con solución de Wisconsin como se indicó anteriormente. El tiempo de isquemia caliente debía ser de alrededor de 15 minutos para que fuera similar al de los experimentos del grupo laparoscópico.

\section{Implante renal}

Posteriormente, tanto en uno como en otro grupo se practicaba:

1. Disección de la vena cava y aorta a nivel inferior al origen de los vasos renales.

2. Clampaje de la vena cava con clamp de satinsky y venotomía con bisturí frío.

3. Anastomosis terminolateral con 2 suturas semicontinuas de prolene de 6 ceros (Fig. 5).

4. Lavado del interior de la anastomosis con solución de heparina al 0,1\% para evitar trombosis.

5. Clampaje con un bulldog de la vena renal, comprobación de la sutura y desclampaje de la vena cava.

6. Clampaje de la aorta y arteriotomía con bisturí frío.

7. Anastomosis terminolateral de la arteria renal con una sutura continua de prolene de 6 ceros.

8. Lavado del interior de la anastomosis con solución de heparina al 0,1\%.

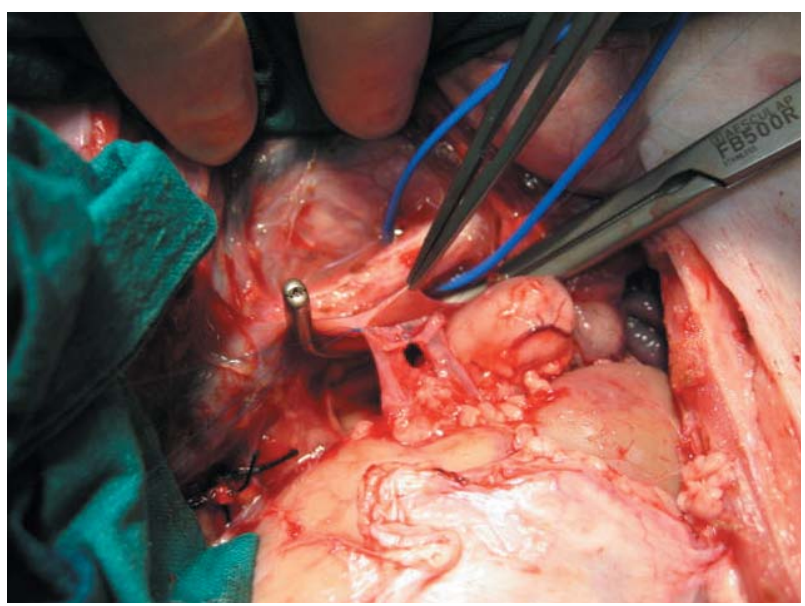

FIGURA 5. Anastomosis venosa en riñón con doble vena. 
9. Colocación de un bulldog a nivel de arteria renal, comprobación de la sutura y desclampaje de la arteria aorta.

10. Segunda biopsia con bisturí frío de unos $4 \mathrm{~mm}$ de largo y 3 de profundidad a nivel de uno de los polos renales.

11. Retirada de los bulldogs y tercera biopsia una vez que el riñón ha quedado correctamente reperfundido.

12. Compresión de las biopsias con un apósito reabsorbible fijado con dos puntos de seda del cero.

13. Cierre de piel y fascias por separado con suturas continuas de seda del cero.

14. Disección de un trayecto subcutáneo desde la incisión paratraqueal hasta la zona dorsal del cuello por donde se hacian pasar las vías centrales hasta el exterior (tunelización). Dichas vias se fijaban a la piel con seda de cero para practicar posteriores extracciones sanguíneas.

15. Cierre de la incisión paratraqueal de la misma forma con seda del cero.

Durante la cirugía se administraba suero Ringer Lactato a dosis de $10 \mathrm{ml} / \mathrm{kg} /$ hora, y antes de finalizar se inyectaba una sobrecarga de $40 \mathrm{mg}$ de seguril para favorecer la diuresis y $1 \mathrm{ml}$ de buprenorfina como analgesia postoperatoria.

Como profilaxis antibiótica se administraba antes de iniciar la intervención $1 \mathrm{~g}$ de cefazolina ev, y en el momento de finalizar una dosis de 15 $\mathrm{mg} / \mathrm{kg}$ de amoxicilina-clavulánico.

Todos los procedimientos quirúrgicos eran llevados a cabo por residentes bajo supervisión de un adjunto.

\section{Derivación urinaria}

Los cerdos hembra eran sondados al inicio de la cirugía con un catéter uretral tipo Bequille de $8 \mathrm{Ch}$, recogiéndose una muestra de orina al inicio y al final de la intervención y retirándose posteriormente la sonda. A los cerdos macho, una vez abierta la laparotomía, se les practicaba una cistostomía con catéter pig-tail de $12 \mathrm{Ch}$ que era fijado a vejiga mediante una bolsa de tabaco con seda de 3 ceros y exteriorizado por contraobertura a la piel del periné, donde se volvía a fijar con un punto simple de seda del cero. Dicha cistotomía se dejaba durante los dias posteriores para la obtención de muestras de orina.

\section{Postoperatorio}

Una vez finalizada la intervención se suspendía la administración de anestésico y se comprobaba el posterior despertar del animal mediante el reflejo podal, esperando la aparición del reflejo de masticación para proceder a la extubación. Una vez extubado se proseguía la ventilación manual con mascarilla de Hall durante unos 20 minutos, tras los cuales el animal era trasladado a la corralina donde se le facilitaba pienso y agua "ad libitum".

Los tiempos de canulación, de anestesia, de la cirugía, de despertar (entre el final de la cirugía y la extubación), de isquemia fría y caliente, así como también de las anastomosis arteriales y venosas se midieron en cada acto quirúrgico. Las complicaciones que se observaron en cada caso fueron igualmente detalladas.

La pauta analgésica en los dias posteriores consistía en buprenorfina intramuscular los 3 primeros dias a dosis de 0,02 $\mathrm{mg} / \mathrm{kg}$. Si se observaban posturas anormales (espalda curvada, inmovilidad...), taquipnea o taquicardia, se administraba una segunda dosis del analgésico.

A las 48 horas se administraba una segunda dosis de amoxicilina-clavulánico, y si el animal presentaba fiebre superior a $38,5^{\circ} \mathrm{C}$ o valores de leucocitosis superiores a $15.000 / \mathrm{mcL}$ se repetía la administración cada 48 horas hasta que cediesen.

El seguimiento del animal se realizaba cada mañana mediante una plantilla preestablecida (Tabla 1).

Si durante el postoperatorio aparecían complicaciones (anuria, fiebre, mal estado general..) se previó prescindir de medidas agresivas y, si las medidas conservadoras no eran suficientes, proceder al sacrificio del animal.

\section{Trasplantectomia}

A la semana del trasplante se repetía el mismo protocolo anestésico y se practicaba la tansplantectomía a través de la misma incisión de laparotomía previa. Una vez el animal quedaba anéfrico se procedia a su sacrificio mediante una sobredosis de pentobarbital (150 mg/kg). Posteriormente el animal era cerrado de nuevo y se practicaban otras técnicas laparoscópicas para la formación de los residentes (colecistectomía, esplenectomía, prostatectomía, cistectomía...) 
Tabla 1. Ficha de seguimiento de los animales de experimentación.

\begin{tabular}{ll}
\hline Aspecto general: & bueno/regular/comatoso \\
Postura: & normal/anormal(encorvado)/tumbado \\
Come: & sí/no \\
Bebe: & sí/no \\
Heces: & sí/no (aspecto) \\
Estado de las suturas: & bueno/regular/malo \\
Hemorragias: & sí/no \\
Vocalizaciones: & no/si (tipo, durante la palpación) \\
Movilidad: & buena/regular/mala \\
Comportamiento ante & \\
estimulos externos: & Alerta/se deja tocar/arreactivo \\
Ta rectal: & normal/hipotermia/hipertermia \\
Tiempo de perfusión gingival: & $<1-2 ” / 1-2 " />1-2 ”$ \\
Color de las mucosas ocular y bucal: & rosadas/blanquecina/violáceas \\
Frecuencia respiratoria: & normal/bradipnea/taquipnea \\
Tipo de respiración: & normal/forzada \\
Ritmo cardiaco: & normal/anormal \\
Observaciones & \\
\hline
\end{tabular}

Si bien los cerdos anestesiados con isofluorano presentaron un despertar más corto que los anestesiados con propofol, esta diferencia no resulta ser significativa en el análisis estadístico.

De los 14 cerdos que no sobrevivieron 11 (78,5\%) fallecieron en el postoperatorio inmediato y los otros $3(21,5 \%)$ entre el segundo y tercer día posterior.

De los primeros, 9 casos fallecieron de forma súbita tras mantener unas constantes correctas durante toda la cirugía después de presentar una taquicardia auricular mantenida que no respondió a fármacos. En dos de estos casos se practicó la autopsia, que reveló en uno la presencia de múltiples focos neumónicos y en otro una pericarditis masiva.

Los resultados descriptivos han sido analizados mediante el programa informático SPSS v 12.0.

\section{RESULTADOS}

Hasta el día de hoy se han llevado a cabo un total de 25 experimentos, de los cuales 7 han sido sometidos a extracción por vía laparoscópica $(28 \%)$ y 18 por vía abierta (72\%). Han sobrevivido hasta el séptimo día 11 de los animales (44\%): 4 de los realizados por vía laparoscópica $(57,14 \%)$ y 7 de los realizados por vía abierta $(38,8 \%)$.

La supervivencia mejora a partir del experimento número 17, en el que se decide trabajar con cerdos macho y practicar la anestesia inhalada (supervivencia del 55,5\% respecto del 37,5\% para el grupo anterior), practicándose en ambos grupos un porcentaje similar de cirugias por vía laparoscópica $(31,2 \%$ en el primero respecto $22,2 \%$ en el segundo).

La media de los tiempos de cada paso de la intervención se pueden ver en la Tabla 2 .

El tiempo medio de despertar pudo ser contabilizado en 16 casos ( 9 cerdos anestesiados con propofol y 7 anestesiados con isofluorano), falleciendo los restantes 9 casos antes de poder ser extubados.
Los otros 2 casos estuvieron relacionados con un sangrado intraoperatorio importante y a una caída accidental del tubo de ventilación.

Tabla 2. Tiempos en minutos referentes a la cirugía experimental

\begin{tabular}{lccc}
\hline Tiempo (minutos) & Media & Rango & DE \\
\hline Canulación & 34 & $15-60$ & 9,6 \\
Anestesia & 273 & $195-390$ & 40,3 \\
Anestesia VL & 278,4 & $240-340$ & 33,5 \\
Anestesia VA & 271 & $195-390$ & 43,4 \\
Cirugia & 196,5 & $105-280$ & 42,7 \\
Cirugia VL & 207,7 & $160-245$ & 26,4 \\
Cirugia VA & 192,1 & $105-280$ & 47,5 \\
Isquemia caliente & 16,9 & $5-25$ & 4,2 \\
Isquemia fría & 100,4 & $60-170$ & 25,5 \\
Sutura venosa & 27 & $10-45$ & 7,7 \\
Sutura arterial & 23 & $15-35$ & 5,8 \\
Despertar & 87,8 & $25-190$ & 50,4 \\
Despertar propofol & 98,9 & $30-190$ & 47,4 \\
Despertar isofluorano & 73,5 & $25-190$ & 54,1 \\
\hline
\end{tabular}

$(\mathrm{VL}=$ vía laparoscópica, $\mathrm{VA}=$ vía abierta) 
Los residentes de nuestro centro han podido realizar un total de 7 nefrectomías laparoscópicas, 37 nefrectomías por vía abierta, 9 trasplantectomias y 22 anastomosis arteriales y venosas, así como múltiples técnicas quirúrgicas por vía laparoscópica una vez el experimento se daba por concluido.

Los gastos que genera un experimento de estas características en nuestro centro se detallan en la Tabla 3.

Tabla 3. Coste aproximado del procedimiento

\begin{tabular}{lr} 
Cerdos $30-35 \mathrm{~kg}$ & $160,50 €$ \\
Transporte & $25,68 €$ \\
Estabulación 7 dias & $15 €$ \\
Residuos & $20 €$ \\
Alquiler quirófano (2 dias) & $360 €$ \\
Total & $581,18 €$ \\
\hline
\end{tabular}

\section{DISCUSIÓN}

\section{E1 modelo porcino}

El cerdo es un animal válido para el ensayo de múltiples procedimientos urológicos como por ejemplo: la cirugía prostática, la nefrectomía parcial, el estudio de diferentes materiales hemostáticos, de los tiempos de isquemia sobre la función renal y el trasplante renal.

La cirugía renal experimental se lleva a cabo preferentemente en modelo porcino por su gran accesibilidad, su semejanza anatómica con el ser humano y el gran tamaño de sus riñones ${ }^{3-9}$. Se han descrito también modelos de abordaje laparoscópico del riñón en conejos y carneros, si bien en el primer grupo el pequeño tamaño de las estructuras anatómicas y del instrumental necesario hacen más dificil el acceso, y en el segundo el uréter, demasiado fino, muchas veces no se puede objetivar durante el acto quirúrgico ${ }^{10}$. Por otra parte el cerdo es de las especies que presenta un indice riñón/peso corporal más alto, lo cual le hace ser más resistente lesiones como la isquemia durante la extracción renal ${ }^{8}$. Estos riñones presentan igualmente una vascularización que, al igual que los humanos, se divide en forma transversal en sus polos craneales y caudales, al contrario que la mayoría de especies, en las que tal división se realiza de forma longitudinal ${ }^{11}$.
Los animales utilizados en nuestros experimentos eran en un principio cerdos de granja hembra, tal como se utilizan en la mayoría de artículos revisados ${ }^{3,4,6-9}$. La literatura los describe como el sexo de elección debido su mayor docilidad y a la mayor facilidad para el sondaje uretral (el meato en las hembras se encuentra en la cara anterior de la vagina, aproximadamente en su tercio interior), mientras que en cerdos macho la forma sinuosa y elongada del pene obliga a cateterizar la uretra a través de una incisión perineal. Además, los cerdos macho presentan un prepucio prominente que se llena de orina y materiales de secreción de las glándulas parauretrales, los cuales pueden contaminar accidentalmente el campo quirúrgico ${ }^{11}$. Sin embargo, los cerdos macho que utilizamos a partir del experimento 17 no presentaron mayores dificultades en su manejo intra y postoperatorio que los cerdos hembra, decidiéndose como técnica para la derivación urinaria la colocación de una cistostomía de la forma anteriormente descrita.

Los cerdos de granja son una especie que, aunque adaptable, es extremadamente sensible al estrés, recomendándose según Kaiser et al. ${ }^{12}$ traerlos desde la granja al estabulario hasta 3 semanas antes del acto quirúrgico para su correcta aclimatación. Este animal presenta un volumen total de sangre que es un $6,5 \%$ del peso corporal (en el hombre es el 7,6\%) pudiéndose extraer hasta un $1 \%$ de una vez y un $7,5 \%$ a lo largo de una semana ${ }^{13}$.

La utilización de otras razas como los minipigs se descartaría como una primera opción dado el menor tamaño de sus estructuras (y por tanto mayor dificultad para el acto quirúrgico) y su mayor coste económico. Sólo estaría indicado su uso cuando se previera un largo tiempo de observación tras la cirugía, en el que los cerdos de granja alcanzarian pesos y tamaños que los harian dificiles de estabular y requeririan de mayor cantidad de comida (un minipig alcanza como máximo los $40 \mathrm{~kg}$, mientras que el cerdo de granja puede llegar a los $300 \mathrm{~kg}$ con dieta de pienso) ${ }^{12}$.

El estabulaje de los cerdos de granja debe realizarse en jaulas de no menos de $3,15 \mathrm{~m}^{2}$ por animal, con una temperatura de entre 10 y $25^{\circ} \mathrm{C}$ para los cerdos adultos y de entre 15 y $32^{\circ} \mathrm{C}$ para aquellos que no hayan alcanzado la madurez. La 
corralina debe presentar como mínimo 10 cambios de aire por hora con un 100\% de aire externo puro y una humedad relativa de entre el $40 \mathrm{y}$ $70 \%{ }^{13}$.

Es importante que el agua sea suministrada mediante un dispositivo que facilite agua corriente con el contacto, ya que estos animales dejan fácilmente de beber de un recipiente donde el agua se haya ensuciado y lo pueden volcar ${ }^{13}$.

Cuando se precise de su inmovilización en la jaula ésta se puede llevar a cabo arrinconándolo en una esquina con la ayuda de un panel de plástico. Si se precisa de una sujeción más firme ésta se llevará a cabo sujetándolo por sus patas traseras. Los procedimientos tales como extracción de sangre o administración de medicamentos intramusculares se puede llevar a cabo mientras el animal esta distraído comiendo ${ }^{13}$.

\section{E1 protocolo anestésico}

Cuando se decide practicar una anestesia mediante agentes endovenosos pueden utilizarse tanto el pentobarbital como la ketamina, la xylacina, la azaperona, el propofol, el midazolam o el fentanilo. La anestesia mediante productos inhalados se puede llevar a cabo con isofluorano, enfluorano, sevofluorano o halotano. Kaiser et al. ${ }^{12}$, tras una experiencia de más de 400 casos, recomiendan para cirugías largas la anestesia inhalada con isofluorano o bien la infusión endovenosa de propofol, midazolam y fentanilo.

Según este grupo el analgésico de elección sería la buprenorfina debido a su larga vida media (sólo requiere de ser administrada 2 ó 3 veces al día). Otras pautas analgésicas aceptadas son la petidina, de vida media corta, la pentazocina, de vida media intermedia, y el metamizol, que a diferencia de los otros dos no puede ser administrado por vía intramuscular y requiere de una vía venosa para su infusión ${ }^{12}$.

Hay autores que defienden la utilización de anestesia intravenosa dado el riesgo que la anestesia inhalada presenta el producir hipertermia maligna en estos animales ${ }^{14}$. Sin embargo, este fenómeno no ha sido observado por nosotros ni descrito en otras series más largas ${ }^{12}$, si bien sí hemos observado que la utilización de anestesia inhalada reduce el tiempo de despertar de estos animales.
Los parches transdérmicos de fentanilo, utilizados ampliamente en pacientes y en muchos experimentos en modelo porcino, no resultan ser de primera elección dada la errática absorción a través de la piel que presenta el fármaco en estos animales ${ }^{7}$.

\section{E1 acto quirúrgico}

Desde que Ratner et al. ${ }^{15}$ llevaran a cabo con éxito la primera extracción renal laparoscópica de donante vivo en 1995, esta técnica se está imponiendo cada vez más como la vía de elección frente la extracción clásica por vía abierta. Los principales inconvenientes que se le atribuian a este abordaje eran la alta frecuencia de lesiones viscerales, la retención de $\mathrm{CO}_{2}$, la aparición de enfisema subcutáneo, la embolia gaseosa y el negativo efecto del neumoperitoneo sobre la función renal ${ }^{16}$. Burgos et al. ${ }^{9}$ señalan como posibles causas de esta oliguria la compresión del parénquima renal, disminución del gasto cardíaco, compresión de la vena cava inferior, obstrucción ureteral y otros factores hormonales asociados. En su serie se compara la extracción renal por vía abierta y por vía laparoscópica en modelo porcino, observándose en este segundo grupo una reducción del flujo arterial renal tanto durante la extracción como una vez el riñón quedaba reperfundido, así como una reducción más lenta de creatinina plasmática durante la primera semana del postoperatorio.

Sin embargo, la serie de Simfoorosh et al. ${ }^{2}$, la primera en la que se practica un estudio randomizado de donantes por vía abierta y laparoscópica, no demostró alteraciones significativas en ambos grupos, en cuanto a complicaciones postoperatorias ni en cuanto a supervivencia del injerto. En cambio, sí se demostró que el grupo de donantes por vía laparoscópica presentaban un menor índice de gasto sanitario y dolor postoperatorio, mejores resultados estéticos y una más rápida incorporación a la actividad cotidiana.

La extracción renal por vía laparoscópica en cerdos había sido ya estudiada por Gill et al. ${ }^{4}$ en 1994, mientras que más recientemente otros grupos como Meraney et al. ${ }^{3}$ o Aguilera et al. ${ }^{6}$ han realizado experimentos en los que practicaban tanto la extracción como el implante por esta vía. Gill et $\mathrm{al}^{4}$ practicaban la nefrectomía contralate- 
ral previa 30 días antes del implante, mientras que Burgos et al. ${ }^{9}$ la realizaban en el mismo momento que el implante y Meraney et al. ${ }^{6}$ la realizaban entre 1 y 4 meses después.

El tiempo quirúrgico medio de Gill et al. ${ }^{4}$ fue de 175 minutos para los experimentos laparoscópicos (DE 60,6) mientras que para los experimentos por vía abierta fue de sólo 72,2 minutos (DE 19,1). En su artículo no indica el tiempo durante el que se practicaba el neumoperitoneo ni la presión de éste. Burgos et al. ${ }^{9}$ fijan en su serie tiempos de neumoperitoneo de 60 minutos, con una presión intraabdominal de $15 \mathrm{~mm} \mathrm{Hg}$.

Los grupos que practican la extracción e implante por vía laparoscópica presentaron lógicamente tiempos quirúrgicos más largos, siendo éstos de 372 minutos (318-474) en el caso de Meraney et al. ${ }^{3}$ y de 480 minutos en el caso de Aguilera et al. ${ }^{6}$.

Nuestra serie presenta tiempos de cirugía por vía laparoscópica semejantes a los que presentaron Gill et al. ${ }^{4}$ hace más de 10 años, si bien con una menor $\mathrm{DE}$, probablemente por haber fijado un mismo tiempo de extracción laparoscópica para todos los experimentos. Los tiempos por vía abierta y laparoscópica son también similares, lo cual facilitará en un futuro comparación del efecto del neumoperitoneo respecto de la extracción vía abierta (Tabla 2).

El tiempo medio de isquemia caliente de Gill et al. ${ }^{4}$ fue de 1,9 minutos en las extracciones por vía abierta (DE 0.6) y de 9,9 minutos en las extracciones laparoscópicas ( $\mathrm{DE}$ de 7,2), asumiendo como tal el intervalo entre que los vasos eran cortados hasta que el injerto era sumergido en hielo. Sin embargo, en este grupo canulación de la arteria renal y posterior perfusión de ringer-lactato a $4^{\circ} \mathrm{C}$, se realizaba aún durante el tiempo laparoscópico a través de una cánula flexible que se introducía por un trocar fino. El tiempo entre la sección de los vasos y la perfusión de esta forma es de 67,5 segundos (25-125 segundos).

Meraney et al. ${ }^{3}$ y Aguilera et al. ${ }^{6}$ practicaban la perfusión mediante la misma técnica laparoscópica con ringer-lactato y solución de Collins a $4^{\circ} \mathrm{C}$ respectivamente, considerando unos tiempos medios de isquemia caliente de 5,1 minutos para el primer grupo y de 5,2 para el segundo.
Burgos et al. ${ }^{9}$ no recogen su tiempo de isquemia caliente, si bien fijan la isquemia fría en 24 horas.

Si comparamos los tiempos que Gill et al. ${ }^{4}$ obtienen en la extracción laparoscópica en cerdos con los tiempos que Simforoosh et al. $^{2}$ obtienen en su serie de 100 casos de extracciones laparoscópicas en pacientes, vemos como los de este último son bastante mayores, con un tiempo quirúrgico de 270,8 minutos (165-490) y un tiempo de isquemia caliente de 8,7 minutos (4-17) ya que, al igual que nosotros, no practicaban la perfusión laparoscópica del riñón con catéter flexible. Es por eso que las diferencias de tiempo de isquemia caliente resultan significativas si se comparan con el mismo de pacientes a los que se les practica el trasplante por vía abierta (1,87 minutos, entre 1 y $5)$, si bien este mayor tiempo de isquemia caliente no influye en la posterior supervivencia del injerto a largo plazo $(93,8 \%$ por vía laparoscópica vs $92,7 \%$ por vía abierta). Estos datos concuerdan con los ya publicados con Buzdon et al. ${ }^{17}$ en 2003, en cuya serie de 640 nefrectomías laparoscópicas de donante vivo no halla una correlación entre un mayor tiempo de isquemia caliente $\mathrm{y}$ niveles séricos de creatinina mayores, ni a corto ni a largo plazo. Dicho autor acepta como correctos tiempos de isquemia caliente de hasta 12 minuto, los más largos que contabilizaron.

Por este motivo en nuestro experimento planteamos un tiempo de isquemia caliente de alrededor de 15 minutos, que por un lado no debía alterar la posterior función del injerto $\mathrm{y}$, por otro, permitía obtener una biopsia del riñón contralateral inmediatamente después de suspender el neumoperitoneo, a fin de conocer con más exactitud el efecto morfológico y funcional que éste condiciona.

La anastomosis se practica en el caso de Gill et al. $^{4}$ a la arteria iliaca externa y vena iliaca común, mientras que los otros dos los anastomosan a los vasos ilíacos externos ${ }^{3,6}$.

Ninguno de los dos grupos enteramente laparoscópicos practican la desinserción del uréter, mientras que Gill et al. ${ }^{4}$ lo liga y posteriormente practica una anastomosis urétero-vesical tipo Gregoir. Ninguno de los cuatro grupos describe complicaciones intraoperatorias, ni tampoco la alta tasa de muertes súbitas intraoperatorias o en el postoperatorio inmediato que nuestra serie presenta $^{3,4,6,9}$. 


\section{E1 postoperatorio}

El protocolo de Gill et al. ${ }^{4}$ preveía el sacrificio del animal a los 30 días del implante y a los 50 días de la nefrectomía contralateral. Los dos casos de Aguilera et al. ${ }^{6}$ eran sacrificados a los 5 días, sin habérseles practicado la nefrectomía contralateral y los 6 cerdos de Meraney et al. ${ }^{3}$ eran sacrificados aleatoriamente entre 1,5 y 5 meses tras el trasplante, después de practicárseles la nefrectomía contralateral también de forma aleatoria entre 1 y 4 meses después. La serie de Burgos et al. ${ }^{9}$ no especifica el momento del sacrificio.

Uno de los animales de Meraney et al. ${ }^{3}$ presentó fiebre en el postoperatorio inmediato y al practicársele el sacrificio se objetivó un riñón trombosado y atrófico. A dicho animal no se le practicó la nefrectomía contralateral. El resto de los 6 animales sobreviven hasta el momento del sacrificio.

Aguilera et al. $^{6}$ por su parte sacrificaba sus dos animales a los 5 días, objetivándose en uno de ellos la trombosis de la arteria iliaca externa y pérdida del injerto.

Gill et al. ${ }^{4}$ describen como complicaciones asociadas a la cirugía sólo dos trombosis venosas en cada grupo (laparoscópico y abierto), si bien su tasa de mortalidad, del $46,6 \%$ es más alta que en los dos grupos anteriores ( 3 de las 5 extracciones por vía abierta y el 4 de las 10 extracciones por vía laparoscópica) en los días posteriores a la cirugía. En el grupo con extracción vía abierta las causas de muerte fueron:

- Neumonía.

- Malfuncionamiento primario del injerto.

- Trombosis del injerto.

En el grupo con extracción por vía laparoscópica fueron:

- Neumonía.

- Gangrena intestinal secundaria a una eventración de la laparotomía.

- Hemorragia por dehiscencia de la sutura venosa.

- Malfuncionamiento primario del injerto.

Burgos et al. ${ }^{9}$ no describen complicaciones postoperatorias ni muertes en su serie.

En nuestra serie si bien el índice de mortalidad era muy alto en los primeros casos, esta mejora sustancialmente con la utilización de cerdos macho y anestesia inhalada, situándola en niveles comparables a los de Gill et al. ${ }^{4}(44,5 \%)$, a pesar de que nuestra serie es la única en que los animales son sometidos a la extracción, implante y nefrectomía contralateral en el mismo acto quirúrgico. Si bien este grupo no indican en ningún caso el momento en que se producen dichas muertes, son debidas en su mayor parte a complicaciones que suceden en los días posteriores a la cirugía, lo cual contrasta con el buen pronóstico que presentan los animales de nuestra serie que sobreviven más allá de las primeras 24 horas.

Otro factor importante del éxito de los experimentos radica en el estado de salud del animal, dado que el mal estado de salud de nuestros cerdos, constatado por las autopsias practicadas, influyó muy posiblemente en los resultados iniciales. Este mal estado de salud fue también constatado por otros centros de nuestra misma área cuyos cerdos eran suministrados por la misma granja. Para mejorar tal situación se puede considerar el cambio de suministrador o demorar los experimentos hasta que nuevas generaciones de animales se vean libres de enfermedad.

\section{La docencia}

Subramonian et $a .^{5}$ ya establecen la cirugía experimental en cerdos como una forma válida y eficaz para el adiestramiento en técnicas laparoscópicas, y gracias a ella practicar procedimientos que posteriormente se llevarán a cabo en humanos. Aguilera et al. ${ }^{6}$ proponen una escala de técnicas quirúrgicas según el grado de dificultad, que comprenderian:

- Nefrectomía radical

- Sutura vesical.

- Disección vascular.

- Nefrectomía parcial.

- Anastomosis vésico-ureteral.

- Colgajo vesical.

- Anastomosis vesico-uretral.

- Neovejiga laparoscópica.

En nuestro centro los residentes han podido realizar igualmente la prostatectomía radical laparoscópica en modelo porcino, siendo importante para esta técnica que se utilicen cerdos sin castrar, pues de lo contrario estos animales presentan atrofia de las vesículas seminales.

Sin embargo, la utilización de animales para estos fines requiere de ciertos principios éticos conocidos como las 3 R: la reducción, el refina- 
miento y el reemplazo. A tal efecto nuestro experimento ha sido concebido con el mínimo número de animales necesarios, velando por su bienestar y correcto mantenimiento, y utilizando métodos alternativos siempre que fuese posible $^{10}$. Es por eso que, previamente a la disección de tejidos y control del sangrado que únicamente se pueden alcanzar hoy en día en animales vivos, nuestros residentes han practicado previamente la coordinación entre la mano y el porta-agujas en el pelvic-trainer y en el simulador digital, con el fin de no alargar demasiado el tiempo de cada técnica y poder realizar el máximo número de ellas con las mayores garantías de éxito.

\section{CONCLUSIONES}

Nuestro protocolo de investigación en cerdos se ha convertido en una herramienta clave para la formación del residente. Este ha demostrado poder realizar todos los pasos de la cirugía sin que esto comprometa la calidad de los resultados obtenidos para la investigación.

La alta tasa de mortalidad de los primeros casos se palió parcialmente utilizando anestesia con gases (que proporcionaba un despertar más rápido $\sin$ otros efectos adversos asociados) y cerdos macho, que no demostraron ser de manejo más dificil que las hembras y permitían practicar la prostatectomía radical laparoscópica una vez finalizado el experimento.

El estado de salud basal de estos animales ha demostrado ser el factor de mal pronóstico más difícil de detectar a priori. La necropsia debería realizarse siempre que fuera posible en los casos de muertes inesperadas y se plantearía, si apareciesen focos infecciosos (p.e. pulmonares), el cambiar de granja suministradora o dejar pasar un tiempo prudencial.

Por último, creemos que los gastos de un experimento de estas características pueden ser más fácilmente asumidos por los centros que dispongan de estabulario.

\section{REFERENCIAS}

1. Oppenheimer F. Trasplante de donante vivo. En: Arias M, Campistol JM, Morales JM. Manual de trasplante renal. Grupo 2 comunicaciones médicas s.1. 2005:257-274.

2. Simforoosh N, Basiri A, Tabibi A, Shakhssalim N, Hosseini Moghaddam SM. Comparison of laparoscopic and open donor nephrectomy: a randomized controlled trial. BJU Int. 2005;95(6):851-855.
3. Meraney AM, Gill IS, Kaouk JH, Skacel M, Sung GT. Laparoscopic renal autotransplantation. J Endourol. 2001; 15(2):143-149.

4. Gill IS, Carbone JM, Clayman RV, Fadden PA, Stone MA, Lucas BA, et al. Laparoscopic live-donor nephrectomy. J Endourol. 1994;8(2):143-148.

5. Subramonian K, DeSylva S, Bishai P, Thompson P, Muir G. Acquiring surgical skills: a comparative study of open versus laparoscopic surgery. Eur Urol. 2004;45(3):346-351.

6. Aguilera Bazan A, Murillo S, Benito de la Vibora J, Cisneros Ledo J, de la Peña Barthel J. Autotrasplante renal laparoscópico experimental. Actas Urol Esp. 2004;28 (1):27-31.

7. Malavasi LM, Augustsson H, Jensen-Waern M, Nyman G. The Effect of Transdermal Delivery of Fentanyl on Activity in Growing Pigs. Acta Vet Scand. 2005;46(3): 149-157.

8. Baldwin DD, Maynes LJ, Berger KA, Desai PJ, Zuppan CW, Zimmerman GJ et al. Laparoscopic warm renal ischemia in the solitary porcine kidney model. Urology. 2004;64(3): 592-597.

9. Burgos FJ, Pascual J, Briones G, Cuevas B, Villafruela J, Correa $\mathrm{C}$ et al. Influence of laparoscopic live donor nephrectomy in ischemia-reperfusion syndrome and renal function after kidney transplantation: an experimental study. Transplant Proc. 2003;35(5):1664-1665.

10. Gaviria F, Uribe C, Escobar F, Castellanos R, Aramburo D, Rodríguez $\mathrm{M}$ et al. Modelo experimental para entrenamiento de nefrectomía laparoscópica. Urología Colombiana. 2005;XIV:63-67.

11. Swindle MM, Smith AC. Information Resources on Swine in Biomedical Research 1999-2000. En: Smith CP, editor. AWIC Resource Series No.11. United States Department of Agriculture. Feb 2000.

12. Kaiser GM, Heuer MM, Fruhauf NR, Kuhne CA, Broelsch $\mathrm{CE}$. General handling and anesthesia for experimental surgery in pigs. J Surg Res. 2006;130(1):73-79.

13. Swindle MM, Smith AC, Laber-Laird K, Dungan L. Swine in Biomedical Research: Management and Models. ILAR J. 1994;36(1).

14. Sáez MJ, Turumbay E, Cilveti MT, Yoldi FJ, Lainez MJ, Martin MP. Anestesia encirugía experimental. Nuestra aportación a lo largo de cuatro años. Anales del sistema sanitario de Navarra. 1999;22(1).

15. Ratner LE, Ciseck LJ, Moore RG, Cigarroa FG, Kaufman HS, Kavoussi LR. Laparoscopic live donor nephrectomy. Transplantation. 1995;60(9):1047-9.

16. Wadström J. Laparoscopic donor nephrectomy technique. Transplant Proc. 2003;35(3):939-940.

17. Buzdon MM, Cho E, Jacobs SC, Jarrell B, Flowers JL. Warm ischemia time does not correlate with recipient graft function in laparoscopic donor nephrectomy. Surg Endosc. 2003;17(5):746-749.

Correspondencia autor: Dr. J.E. Bestard Vallejo

Servicio de Urología

Hospital Universitario Vall d'Hebrón

Passeig de la Vall d'Hebrón, 119-129 - 08035 Barcelona

Tel.: 932746000

E-mail autor: Falta

Información artículo: Original 